\title{
Hydrodynamic analysis of flagellated bacteria swimming in corners of rectangular channels
}

\author{
Henry Shum* \\ Department of Chemical $\&$ Petroleum Engineering, \\ University of Pittsburgh, Pittsburgh, PA 15261, USA \\ Eamonn A. Gaffney ${ }^{\dagger}$ \\ Wolfson Centre for Mathematical Biology, \\ Mathematical Institute, University of Oxford, \\ Oxford OX2 6GG, United Kingdom
}

(Dated: November 15, 2015) 


\begin{abstract}
The influence of nearby solid surfaces on the motility of bacteria is of fundamental importance as these interactions govern the ability of the microorganisms to explore their environment and form sessile colonies. Reducing biofouling in medical implants and controlling the transport of bacterial cells in a microfluidic device are two applications that could benefit from a detailed understanding of swimming in microchannels. In this study, we investigate the self-propelled motion of a model bacterium, driven by rotating a single helical flagellum, in such an environment. In particular, we focus on the corner region of a large channel modelled as two perpendicular sections of no-slip planes joined with a rounded corner. We numerically solve the equations of Stokes flow using the boundary element method to obtain the swimming velocities at different positions and orientations relative to the channel corner. From these velocities, we construct many trajectories to ascertain the general behavior of the swimmers. Considering only hydrodynamic interactions between the bacterium and the channel walls, we show that some swimmers can become trapped near the corner while moving, on average, along the axis of the channel. This result suggests that such bacteria may be found at much higher densities in corners than in other parts of the channel. Another implication is that these corner accumulating bacteria may travel quickly through channels since they are guided directly along the corner and do not turn back or swim transversely across the channel.
\end{abstract}

*phs13@pitt.edu

† gaffney@maths.ox.ac.uk 


\section{INTRODUCTION}

While bacteria cultured in laboratories are generally planktonic and suspended in a homogeneous solution, naturally growing bacterial populations are often found colonizing surfaces and forming complex biofilm communities $[1,2]$. These are problematic for water filtration membranes, medical implants, and other devices. Understanding how bacteria move and interact with surfaces in confined environments is critical for developing strategies to reduce biofouling and also to control the transport of motile cells through lab-on-a-chip microfluidic devices [3], for example. The latter show potential for efficient, high-throughput biological testing and could benefit greatly from controlled manipulation of cells.

Steps toward this objective have already been taken, with many experimental [4-8] and theoretical [9-12] studies exploring the movement of bacteria in microfluidic environments and their ability to colonize microstructured surfaces $[13,14]$. Some demonstrations of applications include harnessing collective effects of swimming bacteria to rotate microfabricated gears $[15,16]$ and fabricating specially designed channels to fractionate a population of bacteria by cell length [3]. Microrobots, including some inspired by the motility of flagellated bacteria, have also been constructed and proposed for biomedical applications as potential carriers for targeted drug delivery or to carry out minimally invasive procedures in a living body [17-19]. These important objectives motivate research in more fundamental aspects of microorganisms interacting with confining surfaces.

It has been shown that bacterial cells, of width $\sim 1 \mu \mathrm{m}$ and with $8 \mu \mathrm{m}$ long flagella, are able to swim through microchannels as narrow as $2 \mu \mathrm{m}$ wide [4]. Remarkably, geometric confinement in such restrictive channels does not significantly slow down the bacteria. In fact, Binz et al. [8] found that swimming speeds of Serratia marcescens were two to three times as high in microchannels $3-10 \mu \mathrm{m}$ wide as the speeds in more open environments, essentially bounded by walls only above and below the cells. Theoretical studies have also indicated that swimming efficiency and speed can be increased by proximity to surfaces $[9,20]$.

Apart from swimming speed, it is important to characterize how trajectories of the organism are affected by surfaces and, hence, where the swimmers tend to be found in relation to the surface. For example, it was observed more than 50 years ago for bull spermatozoa [21], and comparatively recently for bacteria $[6,22]$, that the distribution of swimmers in 
an enclosed sample is not uniform but peaks sharply at the walls of the chamber. Near solid walls, bacteria swim in circular orbits [23, 24]. This phenomenon was also observed in numerical simulations [9] and later explained quantitatively with an analytical model [25]. Consider a bacterium propelled by rotating a right-handed helical flagellum that extends behind the cell body. Viewed from behind, with the wall below the cell, the flagellum rotates clockwise for forward swimming while the cell body rotates counter-clockwise to balance the viscous torque. Since parts of the swimmer closer to the no-slip wall experience higher drag coefficients, the flagellum tends to roll to the right and the cell body rolls to the left, leading to a continual left turn as the bacterium swims.

Understanding of the behavior near a single plane wall guides our intuition but is not enough to completely deduce the motion of microswimers in other confined environments. Various theoretical studies have modelled bacteria, spermatozoa, paramecia, and "squirmer" microswimmers between parallel plates [9, 12, 26], in circular capillary tubes [27-31], and swimming over a backward facing step [32]. In the current study, we examine the motion of a singly flagellated bacterial swimmer in the corner of a long microchannel of rectangular cross-section. We are unaware of prior simulations or theoretical analysis in such a domain, apart from a recent experimental and computational study on artificial helical swimmers driven by a rotating magnetic field [33], and a theoretical study of how an idealized, nonchiral, 2D swimmer is trapped near a corner via the solution of 2D Stokes flow streamfunction equations with singularities representing the swimmer [34].

To restrict the scope of our work, we focus on the region close to one corner of the channel and assume that the cross section is large enough that the other corners and walls may be neglected. We employ the boundary element method to numerically determine the dynamics of two bacterial model swimmers differing only in flagellum length. One is known from prior studies to accumulate at plane boundaries and the other escapes from plane boundaries $[10,12]$. These are the two qualitative behaviors near a single plane wall, excluding collisions, that arise from various combinations of parameters describing the shape of the bacterium.

Using an efficient interpolation technique to simulate many trajectories over long time scales, we assess the general trends of motion near the channel corner for the two bacterial exemplars. We show that the plane boundary accumulating swimmer exhibits corner accumulating behavior. In this case, the model bacterium becomes hydrodynamically bound to 
the corner, swimming in the direction of the channel axis while oscillating laterally along one of the walls of the corner. As expected, no such hydrodynamically bound trajectory is found for the boundary escaping swimmer, which tends to be deflected back into the interior of the channel, away from both walls composing the corner.

The corner accumulation effect we observe is consistent with experiments showing bacteria swimming along one side of a microchannel [8]. Interestingly, it has been found that bacteria do not accumulate in all corners of a channel equally but can be made to show a preference to "swim on the right-hand side" [5]. In our discussion, we suggest a possible explanation for this based on the results of our hydrodynamic simulations.

\section{METHODS}

We model the swimming bacterium as a force-free, torque-free, rigid cell body and helical flagellum as depicted in Fig. 1. The flagellum rotates with a constant angular velocity relative to the cell body, propelling the swimmer. The velocity of the swimmer resulting from this flagellar rotation is determined by solving the equations of Stokes flow with no-slip boundary conditions imposed on the surface of the cell body, flagellum and channel walls; details are given below. Our strategy for analyzing this system is to first use the boundary element method to numerically compute the translational and rotational velocities of the swimmer at different positions and orientations in the channel, and then interpolate between these tabulated values to efficiently construct swimming trajectories and identify steady states and other general behavior.

\section{A. Model bacterium}

We employ the bacterial model used by Shum et al. [10]. The cell body is a prolate spheroid and the right-handed helical shape of the flagellum tapers in amplitude near the cell body so that the base of the filament lies on the axis of the helix, which coincides with the major axis of the body. This model is suitable for bacteria that possess a single flagellum, such as Rhodobacter sphaeroides and Vibrio alginolyticus. For bacteria that grow multiple flagella, such as Escherichia coli, the flagella wrap around the cell body and form a bundle at the rear; this may lead to differences in dynamics in confined spaces. In theoretical 
models, it is common to treat the bundle as a single filament of appropriately adjusted thickness and this approach can yield good agreement with experimental data [25]. We set the characteristic lengthscale for non-dimensionalization to be the volumetric radius of the cell body, $\bar{a}=\left(A_{1} A_{2}^{2}\right)^{1 / 3}$. With average length and width measurements for $R$. sphaeroides [35], this lengthscale is $\bar{a} \approx 0.7 \mu \mathrm{m}$.

(a)

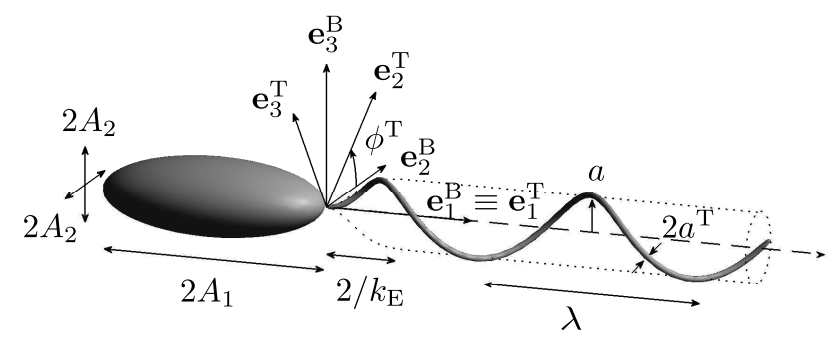

(b)

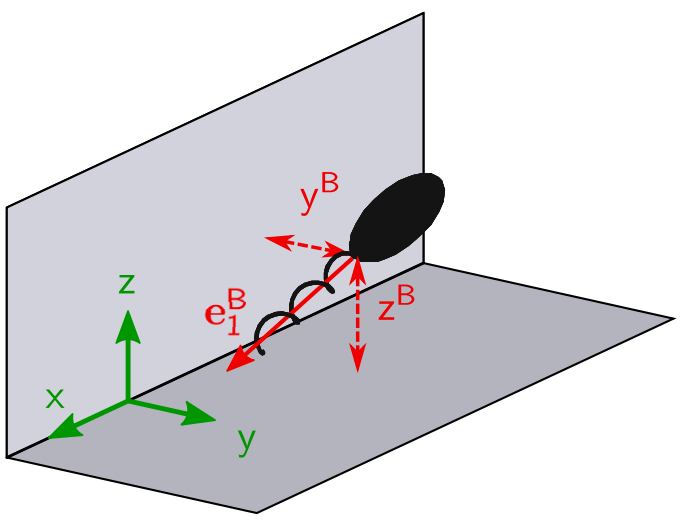

FIG. 1. (Color online) Details of the model bacterium and its configuration in the corner of a large channel. (a) Schematic of model bacterium with labels for orientation vectors and parameters specifying shape. With $\bar{a}=\left(A_{1} A_{2}^{2}\right)^{1 / 3}$ the volumetric radius of the cell body, we use the following parameters for the boundary accumulating swimmer: flagellum length, $L=7.5 \bar{a}$, flagellar wavelength $\lambda=1.8 \bar{a}$, the radius of the helix formed by the flagellum $a=\lambda / 2 \pi$, the flagellar radius, $a^{\mathrm{T}}=0.05 \bar{a}$, and the envelope parameter, dictating how rapidly the helical radius decays on approaching the cell body, $k_{\mathrm{E}}=2 \pi / \lambda$. The boundary escaping swimmer has a shorter flagellum, $L=5 \bar{a}$. Figure reproduced from Shum et al. [12]. (b) A depiction of the swimmer near a channel corner. The velocity of the swimmer, averaged over a revolution of the flagellum, is determined by its position and orientation relative to the walls. Up to symmetry, this configuration is specified by $y^{\mathrm{B}}, z^{\mathrm{B}}$ and $\mathbf{e}_{1}^{\mathrm{B}}$.

We define the swimmer position vector $\mathbf{x}^{\mathrm{B}}=\left(x^{\mathrm{B}}, y^{\mathrm{B}}, z^{\mathrm{B}}\right)^{\mathrm{T}}$ to be the point on the cell body closest to the flagellum. The orientation of the body is described by the set of body directors $\mathbf{e}_{j}^{\mathrm{B}}$ and the tail directors $\mathbf{e}_{j}^{\mathrm{T}}$ are obtained by rotating the body directors through an angle $\phi^{\mathrm{T}}$ about the axis $\mathbf{e}_{1}^{\mathrm{B}}$, which points away from the cell body on its major axis as depicted in Fig. 1. The instantaneous configuration of the swimmer in relation to the stationary channel walls is fully described by $\mathbf{x}^{\mathrm{B}}, \mathbf{e}_{j}^{\mathrm{B}}$ and $\phi^{\mathrm{T}}$. The time evolution of these 
variables is given by

$$
\frac{\mathrm{d}}{\mathrm{d} t} \mathbf{x}^{\mathrm{B}}=\mathbf{U}, \quad \frac{\mathrm{d}}{\mathrm{d} t} \mathbf{e}_{j}^{\mathrm{B}}=\mathbf{\Omega} \times \mathbf{e}_{j}^{\mathrm{B}}, \quad \frac{\mathrm{d}}{\mathrm{d} t} \phi^{\mathrm{T}}=\omega^{\mathrm{M}},
$$

where $\mathbf{U}$ is the translational velocity, $\boldsymbol{\Omega}$ is the rotational velocity of the body, and $\omega^{\mathrm{M}}$ is the angular motor speed.

Since the cell body and flagellum move as rigid bodies, the velocity at a point on the surface of the swimmer can be expressed as

$$
\mathbf{u}(\mathbf{x})= \begin{cases}\mathbf{U}+\boldsymbol{\Omega} \times \tilde{\mathbf{x}}, & \mathbf{x} \in B, \\ \mathbf{U}+\left(\boldsymbol{\Omega}+\omega^{\mathrm{M}} \mathbf{e}_{1}^{\mathrm{B}}\right) \times \tilde{\mathbf{x}}, & \mathbf{x} \in T\end{cases}
$$

where $\tilde{\mathbf{x}}=\mathbf{x}-\mathbf{x}^{\mathrm{B}}$ is the position of the point relative to the reference point, $B$ denotes the surface of the cell body and $T$ denotes the surface of the flagellum. We impose no-slip boundary conditions for the fluid flow field on $B \cup T$ so Eq. (2) also describes the flow field on the surface of the swimmer.

\section{B. Channel walls}

We mesh a finite portion of the intersecting horizontal and vertical walls representing a corner of a large rectangular channel. Since force-free Stokes flows decay spatially at least as quickly as the inverse square of distance, the hydrodynamic effects of the channel walls on the motion of the swimmer are dominated by contributions from the closest parts of the wall. Ramia et al. [9] presented a comparison between using a meshed plate of finite size and using a modified Green's function kernel to satisfy no-slip boundary conditions on an infinite plane wall. For the problems of a spherical particle translating and rotating in a half space, it was shown that the drag forces and torques computed by the two approaches are consistent. This supports our assertion that infinitely large, static boundaries may be truncated to the portion close to the region of interest, i.e., near the swimmer.

The limits of our channel wall mesh are: $|x| / \bar{a} \leq 25, y / \bar{a} \leq 25, z / \bar{a} \leq 25$. In this study, we consider a range of swimmer positions $x^{\mathrm{B}}=0, y^{\mathrm{B}} / \bar{a} \leq 14, z^{\mathrm{B}} / \bar{a} \leq 14$. The meshed portion of the channel walls therefore extends up to a distance of roughly $10 \bar{a}$ around the bacterium.

To avoid discontinuous stresses at the corner $y=z=0$, we connect the vertical and horizontal sections of the wall with a curved corner. In the $y-z$ cross section, this connecting 
section is a quarter circle with radius of curvature $r=\bar{a} / 2$, as illustrated in Fig. 2. The boundary condition for the flow field at a point $\mathbf{x}$ on the combined channel walls is $\mathbf{u}(\mathbf{x})=\mathbf{0}$.

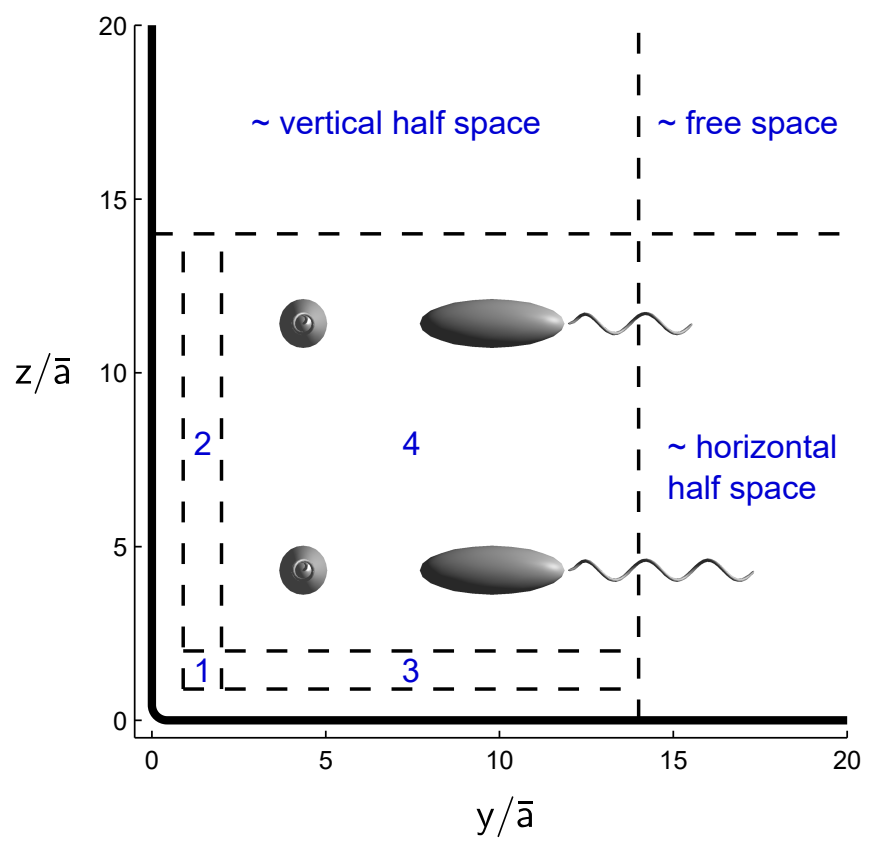

FIG. 2. (Color online) Subdivision of the space near the corner of a large channel. The vertical and horizontal walls at this edge are shown in thick, solid lines. Dashed lines indicate the division of the fluid space into Regions 1-4 and other regions that we do not explicitly analyze. The dynamics in the marked half space and free space regions can be inferred from previous work. The space between the walls and the enumerated regions is largely inaccessible because the finite volume occupied by the swimmer intersects or closely approaches the walls when the junction position $\mathbf{x}^{\mathrm{B}}$ is in this space. The model bacteria are displayed to scale in Region 4 , oriented parallel to the channel $x$-axis (left) and facing towards the vertical wall (right). The swimmers on the top, with shorter flagella, are boundary escapers, and the swimmers on the bottom are boundary accumulators.

We break down the analysis of swimmers in the channel corner by separately considering the dynamics in different regions of space, as detailed in Fig. 2. Previous modelling studies $[10,11,36]$ may be applied to understand the behavior in the regions that are far enough from the corner to be approximated by free space or a half space. Hence, we focus only on the space close to both the horizontal and vertical walls, further subdividing this region in Section III. 


\section{Instantaneous dynamics}

Due to the low Reynolds number associated with bacterial motion, the fluid flow is governed by the equations of unforced, incompressible Stokes flow,

$$
\begin{array}{r}
-\nabla p+\mu \nabla^{2} \mathbf{u}=\mathbf{0}, \\
\nabla \cdot \mathbf{u}=0,
\end{array}
$$

where $p$ is the pressure, $\mu$ is the dynamic viscosity and $\mathbf{u}$ is the velocity field.

The single-layer representation of the boundary integral equation for the velocity field in a domain enclosed by the boundary $\partial V$ is [37]

$$
u_{j}(\mathbf{X})=-\int_{\partial V} q_{i}(\mathbf{x}) G_{i j}(\mathbf{x}, \mathbf{X}) \mathrm{d} S(\mathbf{x})
$$

where the vector field $\mathbf{q}$ is the difference between the surface tractions in the internal and external fluid flow problems and $G_{i j}$ are the $i j$-components of the Stokeslet Green's function defined by

$$
G_{i j}(\mathbf{x}, \mathbf{X})=\frac{1}{8 \pi \mu}\left(\frac{\delta_{i j}}{R}+\frac{R_{i} R_{j}}{R^{3}}\right)
$$

with $\mathbf{R}:=\mathbf{x}-\mathbf{X}$ and $R:=\|\mathbf{R}\|$. As long as the constraint $\int_{\partial V} \mathbf{u} \cdot \mathbf{n} \mathrm{d} S=0$ is satisfied, where $\mathbf{n}$ is the unit normal vector on the domain boundary pointing into the domain, then this representation can be used to describe the flow field exterior to closed surfaces. We consider the fluid domain of interest to be external to the cell body, flagellum, and a large object representing the walls of the channel surrounding the swimmer. The surfaces of these structures are respectively denoted by $B, T$, and $W$. The fluid domain boundary is then $\partial V=B \cup T \cup W$. In the special case that the boundary moves as a rigid body, or as a collection of rigid bodies as in our model system, $\mathbf{q}$ can be replaced by the external traction field $\mathbf{f}$ in the boundary integral equation (Eq. 4) [37]. Note that the wall surface $W$ should, strictly speaking, be closed. As previously discussed, however, accurate dynamics may be computed taking into consideration only those parts of the wall that are close to the swimmer. In practice, we may therefore consider an open wall surface, such as that depicted in Fig. 1(b), provided the wall sections are large enough compared with the swimmer size and the distance from the walls to the swimmer.

As given in the preceding sections, no-slip boundary conditions are used on $\partial V$. We prescribe a constant motor speed $\omega^{\mathrm{M}}$ while the translational and rotational velocities $\mathbf{U}$ and 
$\Omega$ are unknowns to be determined. Specifying the net force and torque on the swimmer allows a unique solution for $\mathbf{U}$ and $\boldsymbol{\Omega}$; we assume the swimmer is force-free and torque-free, which corresponds to the conditions

$$
\int_{B \cup T} \mathbf{f} \mathrm{d} S=\mathbf{0}, \quad \int_{B \cup T} \tilde{\mathbf{x}} \times \mathbf{f} \mathrm{d} S=\mathbf{0} .
$$

A collocation boundary element method (BEM) is used to solve Eq. (4) together with the constraints (6) to obtain the kinematic quantities $\mathbf{U}$ and $\boldsymbol{\Omega}$ describing the motion of the swimmer, as well as the traction distribution $\mathbf{f}$ over fluid domain boundaries. Following the method described by Shum et al. [10], we discretize the boundaries with a mesh of quadratically interpolated triangular elements. The swimmer mesh typically contains $\sim 400$ nodes on the flagellum and $\sim 250$ nodes on the cell body. The channel walls are adaptively refined close to the swimmer and consist of 300-900 mesh nodes.

\section{Phase-averaged dynamical system}

Since many motor revolutions are needed for a bacterium to swim forward by one body length, we assess long timescale dynamics by considering the velocities averaged over a period of rotation. This reduces the dimensionality of phase space and neglects fast oscillations that do not contribute to overall motion. For a given position and body orientation, the phaseaveraged translational velocity is defined by

$$
\begin{aligned}
\overline{\mathbf{U}}\left(\mathbf{x}^{\mathrm{B}}, \mathbf{e}_{1}^{\mathrm{B}}\right) & =\frac{1}{2 \pi} \int_{0}^{2 \pi} \mathbf{U}\left(\mathbf{x}^{\mathrm{B}}, \mathbf{e}_{j}^{\mathrm{B}}, \phi^{\mathrm{T}}\right) \mathrm{d} \phi^{\mathrm{T}} \\
& \approx \frac{1}{N_{\phi}} \sum_{k=1}^{N_{\phi}} \mathbf{U}\left(\mathbf{x}^{\mathrm{B}}, \mathbf{e}_{j}^{\mathrm{B}}, \phi^{\mathrm{T}}=\frac{2 \pi k}{N_{\phi}}\right),
\end{aligned}
$$

where the velocity $\mathbf{U}\left(\mathbf{x}^{\mathrm{B}}, \mathbf{e}_{j}^{\mathrm{B}}, \phi^{\mathrm{T}}\right)$ is computed as described above for position $\mathbf{x}^{\mathrm{B}}$, orientation basis vectors $\mathbf{e}_{j}^{\mathrm{B}}, j=1,2,3$, and motor phase $\phi^{\mathrm{T}}$. Other phase-averaged quantities, such as the mean cell body rotation rate, $\overline{\mathbf{\Omega}}$, are defined analogously. Note that these phase-averaged values are independent of the transverse body directors $\mathbf{e}_{2}^{\mathrm{B}}$ and $\mathbf{e}_{3}^{\mathrm{B}}$ due to axisymmetry of the spheroidal cell body. We repeatedly double the value of $N_{\phi}$ until the resulting change in the computed mean falls below $0.2 \%$; typically this threshold is met with $N_{\phi}=6$ or 12 . In some instances, when the flagellum is very close to the walls, this tolerance is not satisfied even with $N_{\phi}=48$. In these cases we accept an error of up to $5 \%$. 
We consider channels that are uniform and infinite in the $x$-direction. Hence, the instantaneous motion of the swimmer is independent of its $x$-coordinate. Without loss of generality, suppose that $\mathbf{e}_{1}^{\mathrm{B}} \cdot \mathbf{e}_{x} \geq 0$, i.e., the bacterium is swimming with a non-negative component in the negative $x$-direction (the body director $\mathbf{e}_{1}^{\mathrm{B}}$ points backwards). Then, since $\mathbf{e}_{1}^{\mathrm{B}}$ is a unit vector, the orientation is specified by $\eta:=\mathbf{e}_{1}^{\mathrm{B}} \cdot \mathbf{e}_{y}$ and $\zeta:=\mathbf{e}_{1}^{\mathrm{B}} \cdot \mathbf{e}_{z}$. Up to symmetries, the instantaneous configuration of the swimmer is described by $y^{\mathrm{B}}, z^{\mathrm{B}}, \eta$ and $\zeta$. For simplicity, we henceforth drop the superscript B labelling the swimmer position coordinates.

The behavior of the model swimmer in a channel is described by a 4-dimensional system of ordinary differential equations (ODEs) of the form

$$
\begin{aligned}
& \dot{y}(t)=f_{y}(y(t), z(t), \eta(t), \zeta(t)), \\
& \dot{z}(t)=f_{z}(y(t), z(t), \eta(t), \zeta(t)), \\
& \dot{\eta}(t)=f_{\eta}(y(t), z(t), \eta(t), \zeta(t)), \\
& \dot{\zeta}(t)=f_{\zeta}(y(t), z(t), \eta(t), \zeta(t)),
\end{aligned}
$$

where $f_{y}=\bar{U}_{y}, f_{z}=\bar{U}_{z}, f_{\eta}=\left(\overline{\mathbf{\Omega}} \times \mathbf{e}_{1}^{\mathrm{B}}\right) \cdot \mathbf{e}_{y}$ and $f_{\zeta}=\left(\overline{\mathbf{\Omega}} \times \mathbf{e}_{1}^{\mathrm{B}}\right) \cdot \mathbf{e}_{z}$. Motion along the axis of the channel is decoupled from this system but is readily recovered by integrating,

$$
x(t)=x(0)+\int_{0}^{t} f_{x}(y(s), z(s), \eta(s), \zeta(s)) \mathrm{d} s,
$$

where $f_{x}=\bar{U}_{x}$. The functions $f_{x}, f_{y}, f_{z}, f_{\eta}$ and $f_{\zeta}$ vary smoothly with the swimmer configuration and are approximated by linear interpolation from values in look-up tables computed using the boundary element method on a grid of $(y, z, \eta, \zeta)$ phase space. This technique has previously been used for analyzing swimmer motion near infinite plane boundaries [10, 12] and is efficient for generating long trajectories, or many trajectories with different initial conditions, since the computationally intensive BEM is only required for the initial step of constructing the look-up tables.

\section{RESULTS}

We restrict our interest to the region within a distance of around $15 \bar{a}$, corresponding to about $10 \mu \mathrm{m}$ for a bacterium comparable in size to $R$. sphaeroides, from both walls and compute the phase-averaged velocities on a sample of points in $(y, z, \eta, \zeta)$ phase space. Evaluating the dynamics on a regularly spaced grid in parameter space is inefficient because 
the system is much more sensitive to changes in position and orientation when part of the swimmer is very close to a wall. To capture the important dynamics without an excessive number of expensive BEM evaluations, we use locally refined rectangular grids. This is implemented by dividing the channel corner into four regions by proximity to each wall using the following parameter bounds, illustrated in Fig. 2:

Region 1: $0.9 \leq y / \bar{a} \leq 2,0.9 \leq z / \bar{a} \leq 2,-0.2 \leq \eta \leq 0.2,-0.2 \leq \zeta \leq 0.2$

Region 2: $0.9 \leq y / \bar{a} \leq 2,2 \leq z / \bar{a} \leq 14,-0.2 \leq \eta \leq 0.2,-0.6 \leq \zeta \leq 0.6$

Region 3: $2 \leq y / \bar{a} \leq 14,0.9 \leq z / \bar{a} \leq 2,-0.6 \leq \eta \leq 0.6,-0.2 \leq \zeta \leq 0.2$

Region 4: $2 \leq y / \bar{a} \leq 14,2 \leq z / \bar{a} \leq 14,-0.6 \leq \eta \leq 0.6,-0.6 \leq \zeta \leq 0.6$

The bounds on the cell orientation in each region were chosen with consideration to the relatively weak hydrodynamic wall effect on swimmers. A bacterium initially pointing sharply towards a wall will inevitably collide with the wall and a bacterium pointing sharply away from the wall will soon swim out of the region of interest near the wall [10]. Furthermore, bearing in mind that the quantities $y$ and $z$ refer to the position of the cell body-flagellum junction and that the bacterium occupies a finite volume around this point, large inclination angles could entail intersections between the cell body or flagellum and the walls. Therefore, it is unnecessary to consider large angles of orientation towards or away from the walls. Our strategy for analysis is to first consider trajectories in each of the four regions separately before combining the regions to obtain a more complete perspective.

To sample the dynamics in each region, a grid in the 4-dimensional parameter space is constructing using between 7 and 15 values of each configurational parameter within the respective ranges specified above. The BEM computation is performed to determine the swimmer dynamics at each grid point. By interpolating between these phase space evaluation points, we are able to efficiently approximate the ODE system (8). This method is used to simulate a large number of trajectories starting from initial conditions generated in the following manner. For each region, we select each of the region boundaries in turn: top, bottom, left and right, as seen in the $y-z$ plane. This boundary fixes either the starting $y$ or $z$ co-ordinate. The other spatial co-ordinate and the two orientation variables $\eta$ and $\zeta$ are randomly drawn from uniform distributions. To avoid starting too close to the boundary limits in these random variables, we restrict the range of the randomly chosen initial 
conditions to the central half of the intervals defined above for the respective region. For example, a trajectory starting at the top of Region 4 has the initial condition $\left(y_{0}, z_{0}, \eta_{0}, \zeta_{0}\right)$ with $z_{0} / \bar{a}=14$ and the other variables randomly chosen from uniform distributions over the ranges $5 \leq y_{0} / \bar{a} \leq 11$ and $-0.3 \leq \eta_{0}, \zeta_{0} \leq 0.3$.

Since some orientations will cause the bacterium to immediately swim out of the region of interest, and some initial conditions may be invalid due to intersections of the swimmer body with the channel walls, we reject any trajectory that cannot be computed for a duration of at least one motor revolution. We do not consider trajectories starting from the boundaries that are closest to the walls, namely, the left and bottom boundaries of Region 1, the left boundary of Region 2, and the bottom boundary of Region 3. Most trajectories starting from these boundaries are very short due to either the body or flagellum colliding with the wall.

For each region and boundary under consideration, we generate 200 trajectories up to a maximum time of $10^{4} \bar{t}$, where the timescale $\bar{t}=2 \pi / \omega^{\mathrm{M}}$ is the constant period of revolution of the motor. Trajectories may terminate before the prescribed end time if:

1. the trajectory approaches the imposed bounds of the parameter range for its region, or

2. interpolation is not possible due to absence of data.

The first situation indicates that either the swimmer has moved into a different region of the channel or the orientation angle has become too large, which we assume will eventually lead to the bacterium exiting the current region in the direction toward which it has turned.

The second situation occurs because some combinations of the phase space variables correspond to configurations that intersect or come close to intersecting the channel walls. Interpolation is only possible if there are sufficient valid grid points surrounding the desired point. Given a fine enough grid, this will only be a problem when the trajectory approaches too closely to a wall and we therefore treat these events as collisions. In practice, with the grids that were chosen, trajectories sometimes terminate at separation distances of about $0.3 \bar{a}$. This is about half of the semi-minor axis of the cell body and is closer to the wall than typical boundary accumulating orbits [10]. Nevertheless, trajectories that would only transiently attain this proximity may incorrectly be identified as a collision because of this discrete grid effect. 
We analyze how and where each trajectory terminates in order to infer the general or most likely behavior of a swimmer in each of the separate regions. A trajectory is deemed to have exited on the left of a given region if:

1. it approached the lower bound of the $y$ range for the region, the natural definition for leaving a bounded region, or

2. it collided with the left channel wall before reaching the lower bound of the $y$ range, which is possible because of the orientation-dependent, finite $y$-extent of the swimmer, or

3. it approached the upper bound of the $\eta$ range. This condition indicates that the swimmer is turning to strongly face the left wall $\left(\eta \equiv e_{1 y}^{\mathrm{B}}\right.$ increasing $)$ and we assume that this will eventually lead to condition (1) or (2) above.

Corresponding conditions are used to determine instances of exiting through the top, bottom and right of the regions. If a trajectory does not terminate before the prescribed maximum time, the destination is said to be the interior of the region.

Tabulated results of the destinations (boundary of exit or the interior of the region) of trajectories originating from each boundary are given in the Appendix. Note that these statistics should be interpreted with care and only give a qualitative description of the dynamics since the exact distribution of outcomes depends on the chosen parameter bounds, among other factors. Common behavior is determined by examining the tables of trajectory destinations. For each trajectory origin (for example, the top boundary of Region 1), we define the common outcomes to be those that occurred at least $1 / 3$ as many times as the most prevalent destination out of the 200 simulated trajectories. In Fig. 3, we illustrate one trajectory for each common destination of each origin.

Wall effects are expected to be weakest in Region 4 since this is furthest from the walls. Trajectories of both boundary accumulating and boundary escaping swimmers entering Region 4 from the top and right are relatively straight while they are far from the walls, indicating little hydrodynamic deflection of the swimmers. There are, however, clear differences between the accumulators and the escapers. Boundary accumulators are strongly attracted to the two walls. Trajectories starting at the bottom of Region 4 tend to return to this bottom boundary and trajectories starting on the left are unable to traverse the region 
(a)

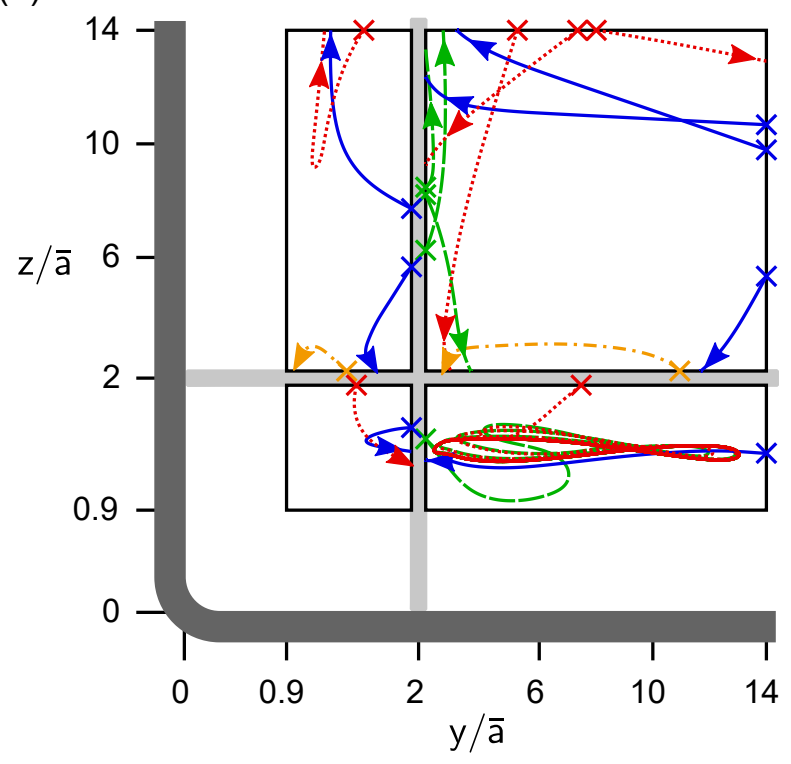

(b)

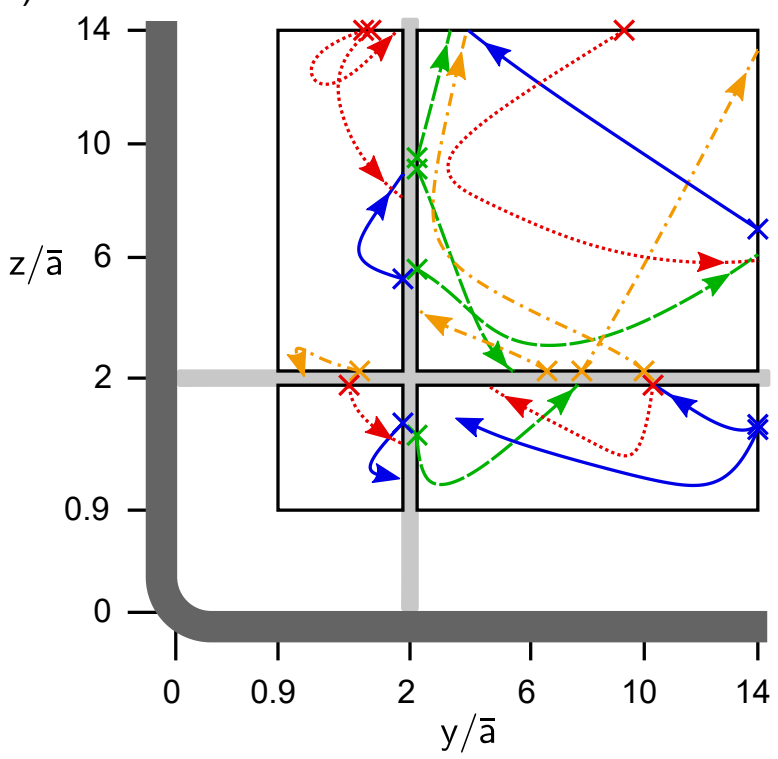

FIG. 3. (Color online) Representative trajectories near a channel corner for (a) boundary accumulating and (b) boundary escaping swimmers. The channel walls are drawn schematically as dark gray strips connected by a rounded corner. For each of the four regions of the corner, delimited by thin, black borders, we show examples of common outcomes for random trajectories entering the region. Starting locations for trajectories are indicated by crosses. Within each region, dashed (green) curves are used for trajectories that start on the left region boundary, dot-dashed (orange) curves start on the bottom, solid (blue) curves start on the right and dotted (red) curves start on the top. Arrows indicate direction of motion along trajectories prior to exiting from the region, whilst the absence of an arrow from a trajectory entails that the bacterium becomes trapped within the respective region. For clarity, the four channel corner regions are drawn slightly separated (by light gray strips) and the axis scales are magnified by a factor of four below the separations at $y / \bar{a}=2$ and $z / \bar{a}=2$, respectively.

to exit on the right. In contrast, boundary escapers starting from either the bottom or the left never turn back toward the originating boundary but commonly exit through any of the other three boundaries. The tendency to escape from boundaries is also evident from the rarity of trajectories starting at the top or right and ending at the bottom or left. Instead, trajectories are generally deflected away as they approach the left and bottom boundaries. 
In Region 1, which is closest to both vertical and horizontal channel walls, we note strong chiral behavior for both boundary accumulators and boundary escapers. The only commonly occurring destination for either swimmer type is the right boundary. This means that there is a clear tendency for the swimmers to follow a counter-clockwise path around the corner, viewed in the $y-z$ plane from behind the swimmer (recall that the orientation is such that the swimmer moves with a negative component in the $x$-direction). This is not obvious from our understanding of bacterial swimmers near plane boundaries. It has been shown that the counter-rotating cell body and flagellum of the swimmer above a no-slip wall lead to a curved path. Our swimmer, which has a right-handed helical flagellum, starting parallel to the channel axis near the bottom, horizontal wall should turn to the left. A swimmer starting near the left, vertical wall should likewise turn to swim upwards. Hence, one might expect a continuity of the behavior, namely, that the swimmer makes a clockwise transition around the corner from the horizontal wall (Region 3) to the vertical wall (Region 2). In fact, none of the simulated trajectories in Region 1 exited at the top (into Region 2).

Above a single, horizontal wall, the boundary accumulator would swim in a circle, continually turning left while remaining near the wall. Neglecting the influence of the vertical channel wall, we would similarly expect boundary accumulators in Region 3 to turn left and swim towards Region 1. As discussed above, however, swimmers in Region 1 have a tendency to migrate into Region 3. This leads to the possibility of either stable or oscillatory motion between Regions 1 and 3. Indeed, the simulated trajectories for the boundary accumulator in Region 3 predominantly remain in the interior of Region 3 until the maximum trajectory duration. This maximum duration was more than 10 times as long as the longest trajectory that eventually exited, suggesting that trajectories remaining in the interior were bound by an attractor within Region 3 rather than simply terminated before escape.

Examining the trajectories that did not exit Region 3, we found that all of these swimmers had become trapped in a unique, figure- 8 shaped periodic orbit in the $y-z$ plane as illustrated in Fig. 4(a). Oscillations in the $y$-direction are much larger than the variations in the vertical direction. Note that while we refer to this as a periodic orbit, it is only the $y-z$ projection of the 3-dimensional path that is periodic, as the swimmer moves monotonically in the $-x$ direction. The orientation deviates only slightly from parallel to the $x$-axis, with vertical (pitch) orientation variable $\zeta \approx-0.05$ so that the tail is always angled toward the lower wall, and the horizontal (yaw) orientation variable $\eta$ oscillating over the range 
$-0.3<\eta<0.3$ (Fig. 4(b)). The $x-y$ projection of trajectories given in Fig. 4(c) represents the top-down view of typical microscopy experiments. In this view, the bacteria are seen swimming primarily along the channel axis with oscillations in distance from the bounding wall.

(a)

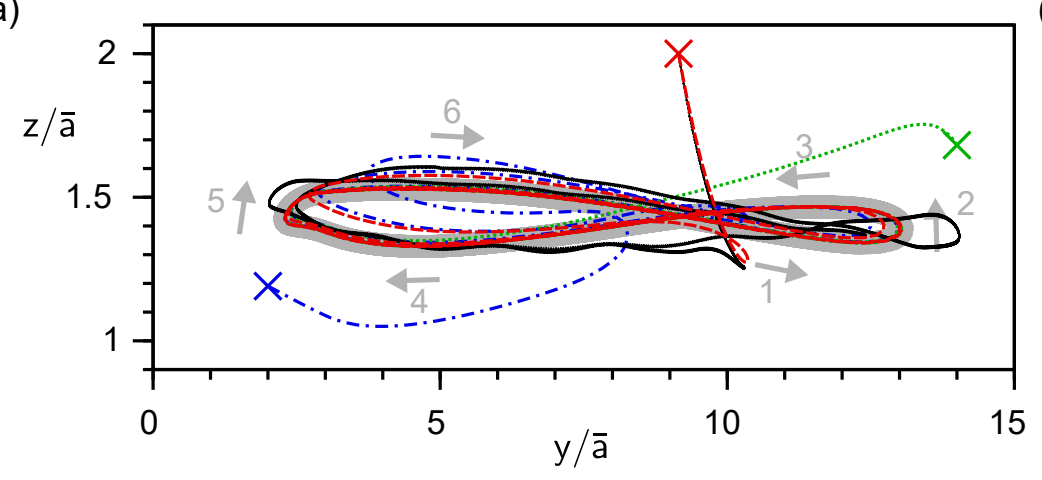

(b)

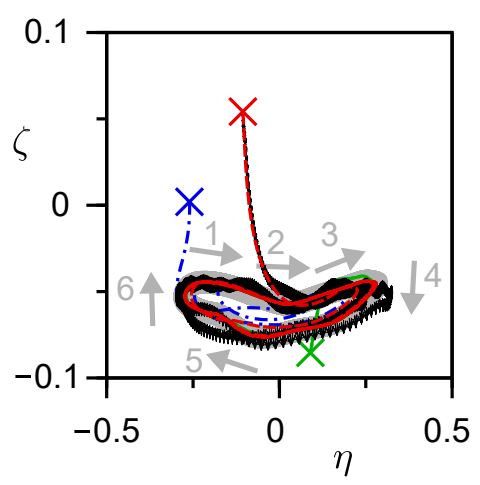

(c)

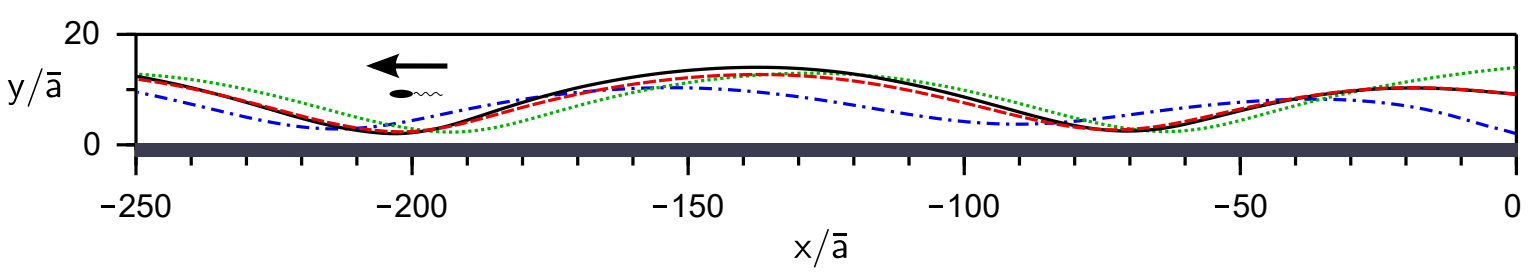

FIG. 4. (Color online) Trajectories of a boundary accumulating bacterium relaxing to a periodic orbit in Region 3 of the channel corner. (a) $y-z$ projections of the trajectories. Three initial conditions, marked by crosses, are used to compute trajectories of the 4-dimensional ODE system (8). All three trajectories converge to the same figure- 8 orbit, plotted with a thick, gray curve. The direction of motion around the orbit is indicated by sequentially numbered arrows. Also shown (solid, black curve) is the trajectory starting from the uppermost initial condition, computed using second order time-stepping with velocities obtained directly by the BEM without phase averaging. The vertical scale is expanded by a factor of five relative to the horizontal scale to magnify the small variations in $z$. (b) $\eta-\zeta$ projections of the trajectories plotted in the same manner as in (a). Positive values of $\eta$ correspond to the bacterium facing the left and positive values of $\zeta$ signify that the swimmer points downward. The numbered points around the periodic orbit correspond to the numbering in (a). (c) The top-down $(x-y)$ view of the trajectories shown in (a) and (b). The $x$ and $y$ axes are set to the same scale and the leftward moving model bacterium is illustrated to scale at around $x / \bar{a}=-200$. 
Having obtained the behavior of the two types of bacterial swimmers in each conceptual region of the corner, we can infer the general dynamics for the corner geometry. For completeness, and in particular, to verify that there are no additional periodic orbits that span more than one region, we continue simulations of all of the previously computed trajectories as they cross from one region to another. As before, the maximum simulation time is set to $10^{4} \bar{t}$. A trajectory terminates if the swimmer approaches the walls or leaves the combined corner region. Using the same criteria as before, we record the final outcome of each trajectory based on the region containing the swimmer immediately prior to termination. The results are listed in Table I. For the boundary accumulator, nearly half of all trajectories entered a stable orbit, persisting until the maximum simulation time. The only such orbit we observed was that shown in Fig. 4. There were no instances of the boundary escaping swimmer remaining inside the combined corner region until the maximum time, confirming the anticipated tendency for the swimmer to escape from corners.

TABLE I. Percentage distributions of final destinations for boundary accumulating and boundary escaping swimmers in combined corner region. The total number of trajectories for each swimmer is 2400. Outcomes correspond to the following: Left - collides with vertical wall; Bottom - collides with horizontal wall; Right - escapes into horizontal half space 3; Top - escapes into vertical half space; Interior - remains in corner region without wall collisions.

\begin{tabular}{c|ccccc} 
& \multicolumn{4}{|c}{ Left Bottom Right } & Top Interior \\
\hline Accumulator & 9 & 14 & 6 & 25 & 46 \\
Escaper & 7 & 4 & 53 & 36 & 0
\end{tabular}

In addition to the analysis of randomly initialized trajectories, we illustrate several trajectories starting from manually specified configurations in Fig. 5. These are consistent with the preceding analysis of individual corner regions. In particular, we note that the boundary accumulator tends to become trapped in a corner accumulating orbit while the boundary escaper is deflected out of the corner region. The chiral bias is also evident in this figure. For the boundary accumulator, this is manifest by the asymmetric position of the periodic orbit, which is along the bottom wall but not the left wall. For the boundary escaper, there is a general tendency to migrate in the counter-clockwise direction close to the corner and all of the presented trajectories exit through the right rather than the top of the corner region. 

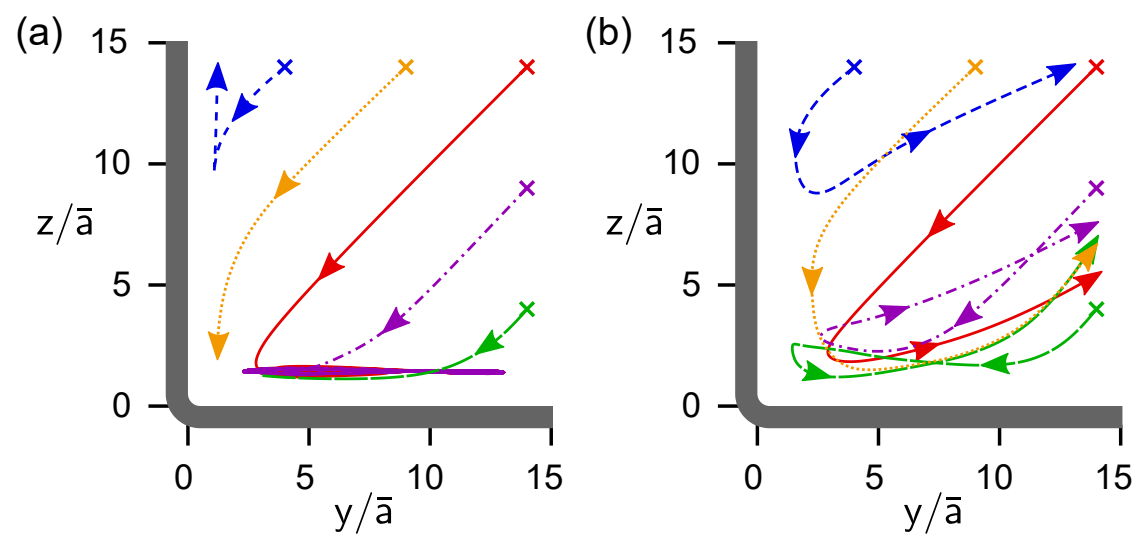

FIG. 5. (Color online) Trajectories of (a) boundary accumulating and (b) boundary escaping bacteria simulated with the interpolation technique in the combined channel corner incorporating Regions 1-4. Five trajectories are shown for each swimmer, starting from the same set of initial positions, marked by crosses. In all cases, the initial orientation was fixed with $\eta_{0}=\zeta_{0}=0.2$ so that the swimmer was pointing obliquely toward the walls. In (a), one trajectory terminates at the arrow closest to the corner due to the swimmer colliding with the lower wall. All other trajectories either escape from the corner region or converge to the corner accumulating orbit.

\section{DISCUSSION}

Experiments by DiLuzio et al. [5] showed that motile E. coli tend to accumulate in the corners of microfluidic channels. Furthermore, the cells were not evenly distributed among all of the corners. Near the top surface, swimmers were found in the corner with the wall on their left. Near the bottom surface, the bacteria swam along the corner with the wall on their right. Different materials used for the top and bottom surfaces caused a preference for swimming at the bottom, resulting in the observation that the bacteria predominantly "swim on the right-hand side". We comment on these findings in light of our simulation results. Note that we use a model bacterium with opposite chirality to that of $E$. coli and should therefore expect our swimmers to "swim on the left-hand side" instead.

Through numerical simulation, we have found that boundary accumulating bacterial swimmers experience a hydrodynamic attraction to corners. Specifically, a stable periodic orbit exists near the corner but the position is not symmetric with respect to the two corner walls. In the bottom left corner, the periodic orbit oscillates horizontally close to the lower wall. Details of this corner accumulating orbit, such as the amplitude of the oscillations 
away from the corner, are likely to vary with geometrical parameters of the swimmer as well as elastic properties of the flagellar filament or hook [10,38]. The location of this attractor, however, corresponds to the trapping along side walls observed experimentally by DiLuzio et al. [5].

Rotating the corner geometry counter-clockwise by $90^{\circ}$, we expect an equivalent periodic attractor in the bottom right corner of the simulated channel, this time oscillating vertically closer to the side wall than to the bottom. Accounting for the opposite chirality, this location corresponds to E. coli swimming in the lower left corner of the channel. Experimentally, this was not observed. The lack of bacteria in this corner could be explained if the bacteria were preferentially attracted to the bottom substrate over the side walls due to differences in the materials. A second possibility is that the small height of the channel used in the experiments interfered with the bottom left attractor, which is at a higher vertical position in the channel than the bottom right attractor and may therefore be more affected by the upper surface. Additional simulations in fully enclosed channels would be necessary to verify this.

Channels of small cross sectional dimensions are known to be effective at guiding the motion of bacteria. If the cells are physically unable to turn back, then they are forced to swim directly along the channel. Moreover, the swimming speeds in narrow channels can be higher than those in less confined environments [8]. Hydrodynamic trapping of bacteria in channel corners means that even if there is sufficient space for the cell to turn, bacteria tend to maintain alignment with the channel. It has been shown that even cells that are able to tumble do not readily escape from surfaces [39]. Hence, we expect that not only small but also large rectangular channels are potentially effective at directing the transport of bacteria. This contrasts with capillary tubes of large circular cross section, which lack corners and allow bacteria to become trapped in circular orbits instead of progressing along the channel [23].

An interesting result from our hydrodynamic simulations is that both our boundary accumulating and escaping bacterial swimmers, which have right-handed helical flagella, tend to move in the counter-clockwise direction around the corner. Viewed from behind, the cell body rotates counter-clockwise as it is propelled and the flagellum rotates clockwise. Above a planar no-slip boundary, this motion would cause the bacterium to turn left and the resulting trajectory would be a counter-clockwise circle when viewed from above [25]. Rolling 
up the planar surface into a cylindrical capillary tube of a certain radius, one might expect the bacterium to trace out a right-handed helical path, circling around the cross-section in the clockwise direction as it progresses along the axis of the tube. While our simulations do not consider fully enclosed channels, the behavior we observe in channel corners suggests motion in the opposite, namely, left-handed, direction.

However, bacterial motility in confined geometries is more complex than motility near plane surfaces. For instance, experiments with bacteria in narrow capillary tubes found that the motion of non-tumbling E. coli cells was consistent with the intuitive expectation, after correcting for the chirality of the flagellum filaments. On the other hand, a different species, Pseudomonas fluorescens, exhibited both left- and right-handed helical trajectories [31]. Like our model swimmer, P. fluorescens has right-handed flagella that rotate clockwise when viewed from behind. Hence, the right-handed helical paths were expected based on the wall effects described above. Left-handed trajectories were hypothesized to result from slip boundary conditions on the capillary walls when the bacteria swam fast enough to exceed a critical shear stress. Our numerical study suggests that hydrodynamic interactions with no-slip boundaries may also lead to left-handed trajectories, though further simulations would be necessary to identify the conditions favoring right- or left-handed helical paths in channels.

\section{CONCLUSION}

Building on well established models and numerical techniques for simulating the propulsion of singly flagellated bacteria in low Reynolds number fluid environments [10, 12, 40], we conducted an analysis of swimming trajectories near the intersection of two mutually perpendicular no-slip walls. This boundary geometry represents the corner of a large channel of rectangular cross section, potentially a conduit for the bacteria in lab-on-a-chip devices, or a crevice that bacteria might encounter in their natural environment. The presence of confining surfaces alters the motion of swimming bacteria and has implications for the migration of these microorganisms.

Previous work has shown that bacterial swimmers can be hydrodynamically attracted to no-slip boundaries [9-11] and that this effect is dependent on parameters describing the

shape of the cell body and flagellum. For the current study, we considered two bacterial 
shapes differing only in the flagellum length. The swimmer with the longer flagellum, which we refer to as the boundary accumulator, has a tendency to swim parallel and close to plane boundaries. The swimmer with the shorter flagellum is a boundary escaper as it tends to swim away from plane boundaries.

As anticipated, trajectories of the boundary escaping swimmer near a channel corner were generally deflected away from the walls and there was no evidence of hydrodynamic trapping of boundary escapers in the corner. In contrast, the plane boundary accumulating swimmer exhibited a stable periodic orbit close to the channel corner with oscillations predominantly along one of the walls due to the chirality of the flagellar rotation. To the best of our knowledge, this is the first theoretical demonstration of hydrodynamic trapping of bacterial microswimmers in channel corners. While attraction to a single plane boundary has previously been explained, it is not obvious a priori that the swimmer would be trapped in a corner where two such boundaries meet. Experiments suggest that bacteria indeed swim preferentially in channel corners, though short range non-hydrodynamic interactions with the surfaces, which were excluded from our model, are also likely to be influential.

These results have a number of implications for practical situations. For example, the attraction of boundary accumulating swimmers to corners of channels indicates that these are the most susceptible areas to biofilm initiation. A circular channel may therefore resist colonization better than square or rectangular channels since there are no corners for bacteria to gather at. Flow through channels is also slowest at the corners due to the no-slip boundary condition. Although we have not investigated the effects of shear flow on bacterial dynamics, it can be hypothesized that corner accumulating bacteria would be less likely to be washed downstream by a background flow through the channel because the no-slip boundary condition weakens the flow at corners where the bacteria tend to swim. On the other hand, it may be possible to design grooved or branching channels that exploit corner accumulation to divert bacteria away from critical zones.

Finally, we note that our results and approach are more generally applicable beyond the focus of bacteria in microchannels. Other types of swimmers, such as spermatozoa, have also been found to swim preferentially in channel corners [41, 42]. Surface topography may therefore play a critical role in the migration of swimming microorganisms in natural environments, such as digestive and reproductive tracts. Our understanding of locomotion in such landscapes could help reduce biofouling or improve designs for controllable nano- 
and microrobots.

\section{Appendix: Tables of trajectory outcomes in corner subregions}

The following tables list the number of occurrences of a particular destination (labeled along the first row) out of 200 simulated trajectories starting from a given origin (labeled in the first column) in different regions of the channel corner. Tables II-V are for the boundary accumulating swimmer and Tables VI-IX are for the boundary escaping swimmer. Entries in boldface are the commonly occurring outcomes (refer to Section III for details).

TABLE II. Origin and destination distributions of boundary accumulator trajectories in Region 1 of channel corners, where boundaries correspond to the following: Left - vertical wall; Bottom horizontal wall; Right - Region 3; Top - Region 2. Interior - remains in Region 1. The left and bottom boundaries are excluded as origins for trajectories.

\begin{tabular}{l|ccccc} 
Origin $\backslash$ Destination & Left & Bottom Right & Top Interior \\
\hline Right & 0 & 0 & $\mathbf{2 0 0}$ & 0 & 0 \\
Top & 7 & 47 & $\mathbf{1 4 6}$ & 0 & 0 \\
\hline Total & 7 & 47 & $\mathbf{3 4 6}$ & 0 & 0
\end{tabular}

TABLE III. Origin and destination distributions of boundary accumulator trajectories in Region 2 of channel corners, where boundaries correspond to the following: Left - vertical wall; Bottom - Region 1; Right - Region 4; Top - vertical half space. Interior - remains in Region 2. The left boundary is excluded as an origin for trajectories.

\begin{tabular}{l|ccccc} 
Origin \Destination & Left & Bottom Right & Top Interior \\
\hline Bottom & 33 & $\mathbf{1 6 7}$ & 0 & 0 & 0 \\
Right & 0 & $\mathbf{6 1}$ & 1 & $\mathbf{1 3 8}$ & 0 \\
Top & 10 & 0 & 0 & $\mathbf{1 9 0}$ & 0 \\
\hline Total & 43 & $\mathbf{2 2 8}$ & 1 & $\mathbf{3 2 8}$ & 0
\end{tabular}


TABLE IV. Origin and destination distributions of boundary accumulator trajectories in Region 3 of channel corners, where boundaries correspond to the following: Left - Region 1; Bottom horizontal wall; Right - horizontal half space; Top - Region 4. Interior - remains in Region 3. The bottom boundary is excluded as an origin for trajectories.

\begin{tabular}{l|ccccc} 
Origin $\backslash$ Destination & \multicolumn{5}{l}{ Left } \\
\hline Left & 0 & 11 & 13 & 0 & $\mathbf{1 7 6}$ \\
Right & $\mathbf{1 4 3}$ & 15 & 0 & 0 & 42 \\
Top & 10 & 0 & 18 & 0 & $\mathbf{1 7 2}$ \\
\hline Total & $\mathbf{1 5 3}$ & 26 & 31 & 0 & $\mathbf{3 9 0}$
\end{tabular}

TABLE V. Origin and destination distributions of boundary accumulator trajectories in Region 4 of channel corners, where boundaries correspond to the following: Left - Region 2; Bottom Region 3; Right - horizontal half space; Top - vertical half space. Interior - remains in Region 4.

\begin{tabular}{|c|c|c|c|c|c|}
\hline Origin \Destination & Left & Bottom & Right & Top & iterior \\
\hline Left & 56 & 62 & 0 & 82 & 0 \\
\hline Bottom & 16 & 160 & 24 & 0 & 0 \\
\hline Right & 44 & 84 & 2 & 70 & 0 \\
\hline Top & 72 & 57 & 60 & 11 & 0 \\
\hline Total & 180 & 363 & 86 & 163 & 0 \\
\hline
\end{tabular}

TABLE VI. Origin and destination distributions of boundary escaper trajectories in Region 1 of channel corners, where boundaries correspond to the following: Left - vertical wall; Bottom horizontal wall; Right - Region 3; Top - Region 2. Interior - remains in Region 1. The left and bottom boundaries are excluded as origins for trajectories.

\begin{tabular}{l|ccccc} 
Origin $\backslash$ Destination & \multicolumn{4}{|c}{ Left Bottom Right } & Top Interior \\
\hline Right & 0 & 5 & $\mathbf{1 9 5}$ & 0 & 0 \\
Top & 14 & 1 & $\mathbf{1 8 5}$ & 0 & 0 \\
\hline Total & 14 & 6 & $\mathbf{3 8 0}$ & 0 & 0
\end{tabular}


TABLE VII. Origin and destination distributions of boundary escaper trajectories in Region 2 of channel corners, where boundaries correspond to the following: Left - vertical wall; Bottom Region 1; Right - Region 4; Top - vertical half space. Interior - remains in Region 2. The left boundary is excluded as an origin for trajectories.

\begin{tabular}{|c|c|c|c|c|c|}
\hline Origin \Destination & Left & 3ottom & Right & Top & terior \\
\hline Bottom & 30 & 160 & 10 & 0 & 0 \\
\hline Right & 0 & 6 & 159 & 35 & 0 \\
\hline Top & 24 & 0 & 77 & 99 & 0 \\
\hline Total & 54 & 166 & 246 & 134 & 0 \\
\hline
\end{tabular}

TABLE VIII. Origin and destination distributions of boundary escaper trajectories in Region 3 of channel corners, where boundaries correspond to the following: Left - Region 1; Bottom horizontal wall; Right - horizontal half space; Top - Region 4. Interior - remains in Region 3. The bottom boundary is excluded as an origin for trajectories.

\begin{tabular}{l|ccccc} 
Origin $\backslash$ Destination & Left & Bottom Right & Top & Interior \\
\hline Left & 0 & 40 & 0 & $\mathbf{1 6 0}$ & 0 \\
Right & $\mathbf{5 8}$ & 34 & 0 & $\mathbf{1 0 8}$ & 0 \\
Top & 9 & 0 & 8 & $\mathbf{1 8 3}$ & 0 \\
\hline Total & 67 & 74 & 8 & $\mathbf{4 5 1}$ & 0
\end{tabular}


TABLE IX. Origin and destination distributions of boundary escaper trajectories in Region 4 of channel corners, where boundaries correspond to the following: Left - Region 2; Bottom - Region 3; Right - horizontal half space; Top - vertical half space. Interior - remains in Region 4.

\begin{tabular}{l|ccccc} 
Origin $\backslash$ Destination & \multicolumn{5}{|c}{ Left } \\
\hline Left & 0 & $\mathbf{3 5}$ & $\mathbf{6 7}$ & $\mathbf{9 8}$ & 0 \\
Bottom & $\mathbf{3 4}$ & 0 & $\mathbf{7 6}$ & $\mathbf{9 0}$ & 0 \\
Right & 18 & 41 & 16 & $\mathbf{1 2 5}$ & 0 \\
Top & 23 & 30 & $\mathbf{1 1 5}$ & 32 & 0 \\
\hline Total & 75 & 106 & $\mathbf{2 7 4}$ & $\mathbf{3 4 5}$ & 0
\end{tabular}

[1] G. O'Toole, H. B. Kaplan, and R. Kolter, Ann. Rev. Microbiod 54, 49 (2000).

[2] T. J. Battin, W. T. Sloan, S. Kjelleberg, H. Daims, I. M. Head, T. P. Curtis, and L. Eberl, Nat. Rev. Microbiod 5, 76 (2007).

[3] S. E. Hulme, W. R. DiLuzio, S. S. Shevkoplyas, L. Turner, M. Mayer, H. C. Berg, and G. M. Whitesides, Lab Chip 8, 1888 (2008).

[4] S. A. Biondi, J. A. Quinn, and H. Goldfine, AلChE.] 44, 1923 (1998).

[5] W. R. DiLuzio, L. Turner, M. Mayer, P. Garstecki, D. B. Weibel, H. C. Berg, and G. M. Whitesides, Nature 435, 1271 (2005).

[6] A. P. Berke, L. Turner, H. C. Berg, and E. Lauga, Phys. Rev. Lett. 101, 038102 (2008).

[7] J. Männik, R. Driessen, P. Galajda, J. E. Keymer, and C. Dekker, Proc.Nat__Acad_Sci._SSA 106, 14861 (2009).

[8] M. Binz, A. P. Lee, C. Edwards, and D. V. Nicolau, Microelectron. Eng. 87, 810 (2010).

[9] M. Ramia, D. Tullock, and N. Phan-Thien, Biophys. J. 65, 755 (1993).

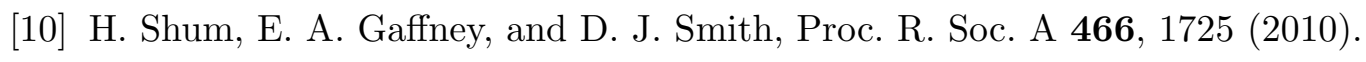

[11] D. Giacché, T. Ishikawa, and T. Yamaguchi, Phys. Rev. E 82, 056309 (2010).

[12] H. Shum and E. A. Gaffney, Phys. Rev. E 91, 033012 (2015).

[13] A. I. Hochbaum and J. Aizenberg, Nano_Lett 10, 3717 (2010). 
[14] R. S. Friedlander, H. Vlamakis, P. Kim, M. Khan, R. Kolter, and J. Aizenberg, Eroc. Natn Acad_Sci._USA 110, 5624 (2013).

[15] A. Sokolov, M. M. Apodaca, B. A. Grzybowski, and I. S. Aranson, Proc. Nat__.Acad.Sci. USA 107, 969 (2010).

[16] R. Di Leonardo, L. Angelani, D. DellArciprete, G. Ruocco, V. Iebba, S. Schippa, M. P. Conte, F. Mecarini, F. D. Angelis, and E. D. Fabrizio, Eroc. Natl.Acad.Sci. USA 107, 9541 (2010).

[17] K. E. Peyer, L. Zhang, and B. J. Nelson, Nanoscale 5, 1259 (2013).

[18] B. J. Nelson, I. K. Kaliakatsos, and J. J. Abbott, Annu. Rev. Biomed. Eng. 12, 55 (2010).

[19] S. Sengupta, M. E. Ibele, and A. Sen, Angew. Chem. Int. Ed. 51, 8434 (2012).

[20] D. F. Katz, L.FluidMech 64, 33 (1974).

[21] Rothschild, ㅁature 200, 381 (1963).

[22] G. Li and J. X. Tang, Phys. Rev. Lett. 103, 078101 (2009).

[23] H. C. Berg and L. Turner, Biophys. J. 58, 919 (1990).

[24] P. D. Frymier, R. M. Ford, H. C. Berg, and P. T. Cummings, Proc. Natl. Acad. Sci. USA 92, 6195 (1995).

[25] E. Lauga, W. R. DiLuzio, G. M. Whitesides, and H. A. Stone, Biophys. J. 90, 400 (2006).

[26] A. Zöttl and H. Stark, Eur. Phys. J. E 36, 4 (2013).

[27] S. Jana, S. H. Um, and S. Jung, Phys. Fluids 24, 041901 (2012).

[28] L. Zhu, E. Lauga, and L. Brandt, L. LFluid_Mech 726, 285 (2013).

[29] B. Liu, K. S. Breuer, and T. R. Powers, Phys. Fluids 26, 011701 (2014).

[30] A. Acemoglu and S. Yesilyurt, Biophys. J. 106, 1537 (2014).

[31] L. Ping, V. Wasnik, and E. Emberly, EEMS Microbio. Ecod 91, 1 (2015).

[32] T. D. Montenegro-Johnson, H. Gadêlha, and D. J. Smith, R. Soc. Open Sci. 2, 140475 (2015).

[33] F. Z. Temel and S. Yesilyurt, Bioinspir. Biomim. 10, 016015 (2015).

[34] A. M. J. Davis and D. G. Crowdy, Eroc. K. Soc. A 468, 3765 (2012).

[35] P. M. Slovak, G. H. Wadhams, and J. P. Armitage, L. Bacteriod 187, 54 (2005).

[36] J. Keller and S. Rubinow, Biophys. J. 16, 151 (1976).

[37] C. Pozrikidis, Boundary integral and singularity methods for linearized viscous flow (Cambridge University Press, New York, 1992).

[38] H. Shum and E. A. Gaffney, Phys. Fluids 24, 061901 (2012).

[39] M. Molaei, M. Barry, R. Stocker, and J. Sheng, Phys. Rev. Lett. 113, 068103 (2014). 
[40] N. Phan-Thien, T. Tran-Cong, and M. Ramia, L.Flwid_Mech 184, 533 (1987).

[41] P. Denissenko, V. Kantsler, D. J. Smith, and J. Kirkman-Brown, एNAS 109, 8007 (2012).

[42] R. Nosrati, P. J. Graham, and D. Sinton, in 4th Micro and Nano Flows Conference (Brunel University London, 2014). 